\title{
Molecular phylogeny and a taxonomic proposal for the genus Streptococcus
}

\author{
F. Póntigo ${ }^{1}$, M. Moraga ${ }^{1,2}$ and S.V. Flores ${ }^{1}$ \\ 'Laboratorio de Antropología, Departamento de Antropología, \\ Facultad de Ciencias Sociales, Universidad de Chile, Santiago, Chile \\ ${ }^{2}$ Programa de Genética Humana, Instituto de Ciencias Biomédicas, \\ Universidad de Chile, Santiago, Chile \\ Corresponding author: S.V. Flores \\ E-mail: sfloresc@uchile.cl
}

Genet. Mol. Res. 14 (3): 10905-10918 (2015)

Received February 4, 2015

Accepted June 17, 2015

Published September 21, 2015

DOI http://dx.doi.org/10.4238/2015.September.21.1

\begin{abstract}
Alternative phylogenies for the genus Streptococcus have been proposed due to uncertainty about the among-species group relationships. Here, we performed a phylogenetic analysis of the genus Streptococcus, considering all the species groups and also the genomic data accumulated by other studies. Seventy-five species were subjected to a Bayesian phylogenetic analysis using sequences from eight genes (16S rRNA, rpoB, sodA, tuf, rnpB, gyrB, dnaJ, and recN). On the basis of our results, we propose a new Phylogeny for the genus, with special emphasis on the inter-species group level. This new phylogeny differs from those suggested previously. From topological and evolutionary distance criteria, we propose that gordonii, pluranimalium, and sobrinus should be considered as new species groups, in addition to the currently recognized groups of mutans, bovis, pyogenic, suis, mitis, and salivarius.
\end{abstract}

Key words: Bayesian analysis; Phylogeny; Streptococcus;

Species group 


\section{INTRODUCTION}

The genus Streptococcus is a diverse lineage belonging to the lactic acid group of bacteria. Current taxonomy places this genus, as well as the genus Lactococcus, within the family Streptococcaceae in the order Lactobaillales (Facklam, 2002). These organisms are Gram-positive, spherical, and catalase-negative, and many are facultative anaerobes (Montes and García-Arenzana, 2007; Lal et al., 2011).

Recent molecular systematic studies based on 16S rRNA sequences have provided evidence to subdivide Streptococcus into six "species groups": pyogenes, anginosus, mitis, salivarius, bovis, and mutans (Kawamura et al., 1995; Facklam, 2002; Rodicio and Mendoza, 2004; Montes and García-Arenzana, 2007; Lal et al., 2011).

In addition to the striking phylogenetic diversification, the ecological features of the members of the Streptococcus genus encompass a myriad of environments and life styles. While some species are well known for their clinical importance as pathogens, others have been characterized as members of a range of normal microbiomes in different anatomical structures in animals and humans. This distribution pattern explains their regular occurrence in contaminated biological samples (Montes and García-Arenzana, 2007; Glazunova et al., 2010; Boggs et al., 2012).

The features of pathogenicity associated with the genus Streptococcus are also diverse: meningitis, pneumonia, endocarditis, fasciitis, and dental caries are among the better known conditions (Glazunova et al., 2010). Normal human reservoirs of Streptococcus include different compartments of the oral cavity and skin, and the respiratory, digestive, gastric, and urinary tracts (Hardie and Whiley, 1997). However, the complete home range for most species of the genus is largely uncertain since this knowledge depends on sampling strategies that are not normally focused on revealing species habitats.

Systematic studies have been performed in the genus. Kawamura et al. (1995) carried out a pioneer study that proposed the current general phylogenetic organization for the genus Streptococcus. In that study, 16S rRNA gene sequences from 28 distinct species were analyzed leading to a hypothesis of six species groups that is still generally accepted.

Simmon et al. (2008) examined the viridans group using $16 \mathrm{~S}$ rRNA, tuf, and rpoB gene sequences from 22 species and subspecies to evaluate phylogenetic relationships within the genus in a study of samples from patients affected by endocarditis. They demonstrated horizontal gene transfer and different rates of molecular substitutions among DNA fragments in the genus, making phylogenetic inferences a challenging subject for this lineage.

Furthermore, other studies have evaluated the phylogenetic certainty of different gene fragments. For example, Täpp et al. (2003) analyzed the gene rnpB in 50 species, in order to obtain phylogenetic information from a source other than 16S rRNA. Similarly, Itoh et al. (2006) proposed the $d n a J$ and $g y r B$ genes as good phylogenetic markers, and analyzed 45 species and subspecies. Additionally, the $\operatorname{recN}$ gene has been shown to render statistically confident clades in an extensive analysis that included 60 species (Glazunova et al., 2010).

To date, the phylogenetic relationships among the known species groups have not been resolved. For example, Itoh et al. (2006) concluded that disagreements between the trees obtained from three different gene fragments could be explained either by horizontal gene transfer or different mutation rates. This also can be seen in the phylogenetic results of Simmon et al. (2008) who described phylogenetic discordances that could be explained 
by horizontal gene transfer. In both studies, the analyses were performed using phylogenetic distances; the effects of different phylogenetic methods were not evaluated. Glazunova et al. (2006) and Täpp et al. (2003) used diverse genes and different phylogenetic approaches (distances and Bayesian analysis, respectively) and produced two alternative phylogenetic hypotheses (Figure 1).

(A)

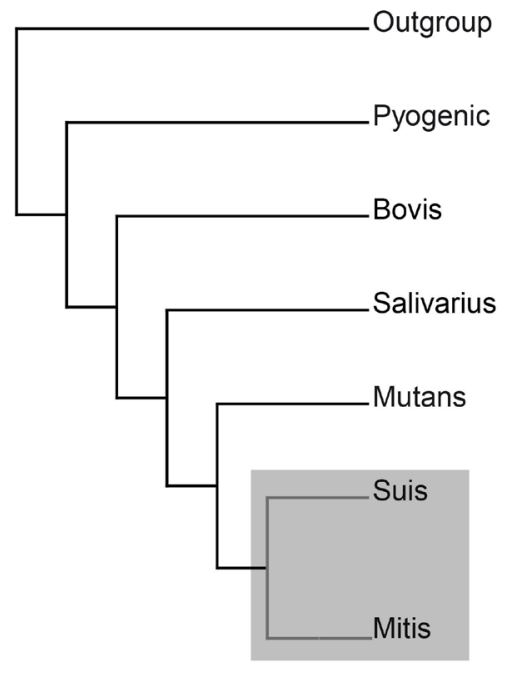

(B)

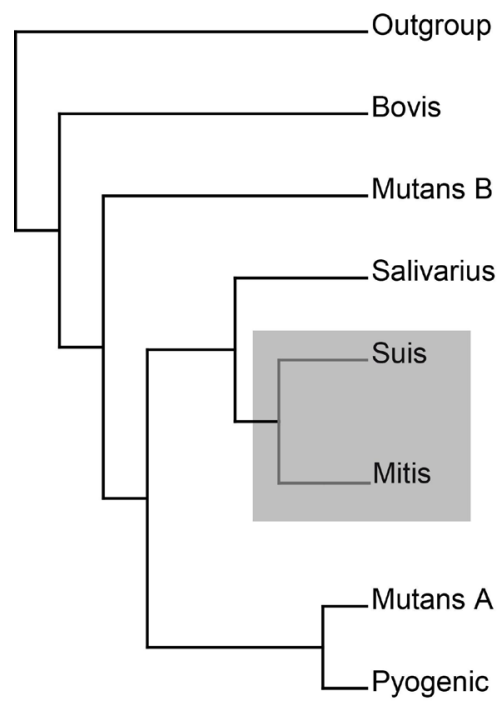

Figure 1. Phylogenetic hypothesis by (A) Glazunova et al. (2006, Figure 1), based on fragment sequences of recN gene; and (B) Tapp et al. (2003; Figure 3), using sequences of 16S r RNA and rnpB. 
As shown in Figure 1, the species groups suis and mitis are in sister clades in both topologies. This is the only convergence between these phylogenetic hypotheses, as other relationships remained unclear.

Using the available genomic databases it is possible to incorporate all the gene fragment data that has been reported into a single phylogenetic analysis. This approach will enable the development of a new systematic work on this genus, covering all the known species groups. In this article, we attempt to create an extensive phylogeny for the genus Streptococcus, and contrast our proposed phylogeny with previously published hypotheses.

\section{MATERIAL AND METHODS}

\section{Samples}

DNA sequences from the genes $\operatorname{sod} A$, tuf, rpoB, 16s rRNA, rnpB, dnaJ, gyrB, and recN were retrieved from GenBank (www.ncbi.nlm.nih.gov/genbank; Table 1). Seventyfive species and subspecies of Streptococcus were analyzed. Lactococcus lactis subsp lactis and Lactococcus lactis subsp cremoris were selected as outgroups, since this genus is one of the four members of the family Streptococcaceae. Although a primary analysis showed that Pilibacter is the sister clade of Streptococcus, the lack of data for this genus did not allow robust analyses to be performed (Figure 2).

\section{Molecular phylogenetic analyses}

Nucleotide sequences were aligned using the software BioEdit V. 7.0.9.0 (Hall, 1999). The alignments were checked and re-edited by eye. In order to avoid the effect of saturation of the phylogenetic signal, especially due to synonymous substitutions, amino acid sequences were also used for the six protein coding genes $(\operatorname{sod} A, \operatorname{tuf}, \operatorname{rpoB}, \operatorname{dnaJ}, \operatorname{gyr} B$, and $\operatorname{rec} N)$. The non-coding genes 16s rRNA and rnpB were included in both analyses of nucleotide sequences and amino acid derived sequences. Both nucleotide and amino acid derived sequences were concatenated in the same super-matrix. Absent data were coded as missing data and incorporated in subsequent analyses. This "super-matrix" approach has been shown to perform better than eliminating data, or using non-concatenated matrices, especially when Bayesian methods are used for phylogenetic inference (Wiens and Moen, 2008).

Models of molecular evolution for nucleotide sequences were selected using the Akaike information criterion test (AIC), as implemented in the software Modeltest (Posada and Crandall, 1998) and MrModeltest (Nylander, 2004). Models for amino acid sequences were selected using the PROTTEST v. 3.0 software (Abascal et al., 2005).

Bayesian inference on the complete super matrix was performed using Mr. Bayes v. 3.0b4, specifying four million generations and four independent chains. The resulting tree identified seven subclades that were used as the basis for subsequent analyses (Figure 3 and Table 2).

In order to solve phylogenetic relationships among species groups, a new matrix was built sampling two species belonging to each of the groups. This matrix, called the "minimatrix", was used for Bayesian inference using the same conditions and model substitutions as described above for the complete super-matrix. Phylogenetic concordance among the different DNA sequences was assessed by the "homogeneity test" (Farris et al., 1994, 1995) as implemented in Paup, in order to evaluate the phylogenetic compatibility among gene fragments inside the super-matrix. 
Table 1. List of species, accession numbers and size (bp) of each gene fragment.

\begin{tabular}{|c|c|c|c|c|c|c|c|c|}
\hline \multirow[t]{2}{*}{ Species } & \multicolumn{2}{|r|}{$\operatorname{sod} A$} & \multicolumn{2}{|r|}{ tuf } & \multicolumn{2}{|r|}{ rров } & \multicolumn{2}{|r|}{ recN } \\
\hline & bp & Accession No. & $\mathrm{bp}$ & Accession No. & bp & Accession No. & $\mathrm{bp}$ & Accession No. \\
\hline S. mutans & 612 & AE014133.2 & 1197 & AE014133.2 & 3105 & AP010655.1 & 1249 & EU917289.1 \\
\hline S. agalactiae & 609 & AE009948.1 & 1197 & AL766847.1 & 3105 & AE009948.1 & 1249 & EU917242.1 \\
\hline S. acidominimus & 435 & Z95892.1 & 761 & AY266992.1 & 691 & AF535181.1 & 1249 & EU917241.1 \\
\hline S. anginosus & 453 & FJ712177.1 & 826 & AF276257.1 & 3105 & AF535183.1 & 1249 & EU917248.1 \\
\hline S. alactolyticus & 435 & AJ297185.1 & - & - & 680 & DQ232445.1 & 1249 & EU917226.1 \\
\hline S. australis & 435 & GU907529.1 & 761 & EU156907.1 & 680 & DQ132983.1 & - & - \\
\hline S. caballi & 399 & EF364100.1 & - & - & - & - & - & - \\
\hline S. canis & 435 & Z99175.1 & 761 & EU156908.1 & 680 & DQ232488.1 & - & - \\
\hline S. castoreus & 435 & EU003820.1 & - & - & 680 & EU003817.1 & 1249 & EU917312.1 \\
\hline S. criceti & 435 & Z95898.1 & 821 & AF276260.1 & 680 & DQ232485.1 & 1249 & EU917257.1 \\
\hline S. cristatus & 435 & GU907530.1 & 761 & AF276261.2 & 2061 & $\begin{array}{l}\text { AF194513.1, } \\
\text { AF194514.1, } \\
\text { AB199920.1 }\end{array}$ & 1249 & EU917258.1 \\
\hline S. devriesei & 435 & DQ232544.1 & - & - & 680 & DQ232446.1 & 1249 & EU917306.1 \\
\hline S. didelphis & 435 & DQ232545.1 & - & - & 680 & DQ232447.1 & 1249 & EU917259.1 \\
\hline S. downei & 435 & Z95899.1 & 792 & AF276262.1 & 680 & DQ132984.1 & - & - \\
\hline S. dysgalactiae subsp equisimilis & 486 & AP010935.1 & 1215 & AP010935.1 & 3105 & AP010935.1 & 1249 & EU917227.1 \\
\hline S. constellatus & 453 & FJ712176.1 & 821 & AF276259.1 & 680 & FJ712146.1 & 1093 & FJ712113.1 \\
\hline S. intermedius & 453 & FJ712187.1 & 799 & AF276267.1 & - & - & 1249 & EU917282.1 \\
\hline S. entericus & 435 & DQ232547.1 & - & - & 680 & DQ232448.1 & 1249 & EU917228.1 \\
\hline S. equi subsp. equi & 486 & FM204883.1 & 1100 & FM204883.1 & 3105 & FM204883.1 & 1249 & EU917229.1 \\
\hline S. equinus & 435 & AJ297213.1 & 792 & AF276258.1 & 3105 & AF535187.1 & 1249 & EU917252.1 \\
\hline S. ferus & 435 & DQ132986.1 & 792 & AF276265.1 & 686 & AY770000.1 & 1249 & EU917265.1 \\
\hline S. gallinaceus & 435 & DQ232548.1 & - & - & 680 & DQ232503.1 & 1246 & EU917266.1 \\
\hline S. gallolyticus & 486 & FN597254.1 & 1100 & FN597254.1 & 3105 & FN597254.1 & - & - \\
\hline S. gordonii str. Challis substr. $\mathrm{CH} 1$ & 486 & СР000725.1 & 1100 & СР000725.1 & 3105 & СР000725.1 & - & - \\
\hline S. halichoeri & 435 & DQ232571.1 & - & - & 680 & DQ232471.1 & 1249 & EU917277.1 \\
\hline S. henryi & 399 & EF364099.1 & - & - & - & - & - & - \\
\hline S. hyointestinalis & 435 & DQ232550.1 & - & - & 680 & DQ232449.1 & 1249 & EU917231.1 \\
\hline S. hyovaginalis & 435 & DQ232551.1 & - & - & 680 & DQ232450.1 & 1249 & EU917232.1 \\
\hline S. infantarius subsp infantarius & 435 & AJ306980.1 & 761 & EU156915.1 & 672 & EU420169.1 & - & - \\
\hline S. infantis & 432 & GU907532.1 & 761 & EU156917.1 & 680 & DQ232482.1 & 1249 & EU917280.1 \\
\hline S. iniae & 486 & EU661272.1 & 761 & EU156918.1 & 680 & DQ232493.1 & 1249 & EU917281.1 \\
\hline S. luteciae & 435 & AJ297212.1 & - & - & - & - & & \\
\hline S. lutetiensis & 426 & GU991740.1 & 761 & EU156916.1 & 680 & DQ232480.1 & 1249 & EU917279.1 \\
\hline S. macacae & 435 & DQ232553.1 & 792 & AF276268.1 & 680 & DQ232452.1 & 1249 & EU917285.1 \\
\hline S. macedonicus & 435 & AJ297187.1| & 761 & EU156913.1 & 693 & AY315156.1 & 1249 & HE613569.1 \\
\hline S. marimammalium & 435 & EU003821.1 & - & - & 659 & EU003818.1 & 1249 & EU917313.1 \\
\hline S. massiliensis & 451 & AY769999.1 & 761 & EU156919.1 & 691 & AY769998.1 & 1249 & EU917311.1 \\
\hline S. minor & 435 & DQ232572.1 & - & - & 680 & DQ232472.1 & 1249 & EU917286.1 \\
\hline S. mitis & 486 & FN568063.1 & 1100 & FN568063.1 & 3105 & FN568063.1 & 1249 & EU917233.1 \\
\hline S. oligofermentans & 435 & DQ232554.1 & 761 & EU156926.1 & 680 & DQ232508.1 & 1249 & EU917290.1 \\
\hline S. oralis & 435 & DQ232576.1 & 764 & AF276270.1 & 2658 & $\begin{array}{l}\text { AY695496.1, } \\
\text { AB199956.1 }\end{array}$ & 1249 & EU917234.1 \\
\hline S. orisratti & 435 & DQ232555.1 & - & - & 680 & DQ232453.1 & 1249 & EU917235.1 \\
\hline S. ovis & 435 & DQ232556.1 & - & - & 680 & DQ232454.1 & 1249 & EU917236.1 \\
\hline S. parasanguinis & 435 & GU907536.1 & 789 & AF276271.1 & - & - & 1249 & СР002843.1 \\
\hline S. parauberis & 435 & AJ544723.1 & 761 & EU156937.1 & 680 & DQ232455.1 & 1212 & EU917293.1 \\
\hline S. pasteurianus & 435 & DQ232583.1 & 761 & EU156914.1 & 680 & DQ232505.1 & 1249 & EU917273.1 \\
\hline S. peroris & 435 & DQ232541.1 & 761 & EU156938.1 & 680 & DQ232483.1 & 1249 & EU917294.1 \\
\hline S. phocae & 435 & AJ547799.1 & - & - & 680 & DQ232456.1 & 1249 & EU917237.1 \\
\hline S. pluranimalium & 435 & DQ232557.1 & 761 & EU156939.1 & 680 & DQ232457.1 & 1249 & EU917295.1 \\
\hline S. plurextorum & 356 & AM774231.1 & - & - & 687 & AM774232.1 & - & - \\
\hline S. pneumoniae & 486 & FM211187.1 & 1100 & FM211187.1 & 3105 & FM211187.1 & 1249 & EU917238.1 \\
\hline S. porcinus & 435 & Z99177.1 & - & - & 680 & DQ232486.1 & 1249 & EU917296.1 \\
\hline S. pseudopneumoniae & 390 & AB200048.1 & 761 & EU156954.1 & 680 & EU003819.1 & 1249 & EU917314.1 \\
\hline S. pyogenes & 486 & СР000262.1 & 1100 & AE009949.1 & 3105 & СР000261.1 & 1249 & EU917297.1 \\
\hline S. ratti & 435 & DQ232559.1 & 792 & AF276272.1 & 680 & DQ232484.1 & 1249 & EU917298.1 \\
\hline S. salivarius & 432 & GU907538.1 & 792 & AF276273.1 & 691 & AF535169.1 & 1249 & EU917239.1 \\
\hline S. sanguinis & 486 & СР000387.1 & 1100 & СР000387.1 & 2922 & СР000387.1 & 1249 & EU917299.1 \\
\hline
\end{tabular}

Continued on next page 


\begin{tabular}{|c|c|c|c|c|c|c|c|c|}
\hline \multirow[t]{2}{*}{ Species } & \multicolumn{2}{|r|}{$\operatorname{sod} A$} & \multicolumn{2}{|r|}{ tuf } & \multicolumn{2}{|r|}{ rpoB } & \multicolumn{2}{|r|}{$\operatorname{rec} N$} \\
\hline & bp & Accession No. & bp & Accession No. & bp & Accession No. & bp & Accession No. \\
\hline S. sinensis & 435 & DQ232560.1 & 761 & EU156962.1 & 680 & DQ232458.1 & 1249 & EU917305.1 \\
\hline S. sobrinus & 435 & Z95919.1 & 780 & AF276275.1 & 680 & DQ232489.1 & - & - \\
\hline S. suis & 486 & СР000408.1 & 1100 & СР000408.1 & 3102 & AM946016.1 & 1249 & СР002570.1 \\
\hline S. thermophilus & 486 & СР000024.1 & 1100 & СР000024.1 & 3105 & СР000024.1 & 1249 & EU917300.1 \\
\hline S. thoraltensis & 435 & DQ232562.1 & - & - & 680 & DQ232461.1 & 1249 & EU917303.1 \\
\hline S. uberis & 486 & AM946015.1 & 1100 & AM946015.1 & 3105 & AM946015.1 & 1249 & EU917309.1 \\
\hline S. urinalis & 435 & DQ232561.1 & 761 & EU156963.1 & 680 & DQ232460.1 & 1249 & EU917240.1 \\
\hline S. vestibularis & 436 & AY687381.1 & 781 & AF 276277.1 & 686 & AY 687377.1 & 1249 & EU917304.1 \\
\hline L. lactis subsp lactis & 481 & СР001834.1 & 1084 & СР001834.1 & 3105 & СР001834.1 & - & - \\
\hline L. lactis subsp cremoris & 467 & СР000425.1 & 1084 & СР000425.1 & 3105 & СР000425.1 & - & - \\
\hline \multirow[t]{2}{*}{ Species } & \multicolumn{2}{|r|}{$16 S r R N A$} & \multicolumn{2}{|r|}{$r n p B$} & \multicolumn{2}{|r|}{ dnaJ } & \multicolumn{2}{|r|}{ gyrB } \\
\hline & $\mathrm{bp}$ & Accession No. & bp & Accession No. & bp & Accession No. & bp & Accession No. \\
\hline S. mutans & 1542 & AB294730.1 & 380 & AJ511678.1 & 1134 & AE014133.2 & 1953 & AP010655.1 \\
\hline S. agalactiae & 1537 & AF459432.1 & 374 & AJ511673.1 & 1140 & AL766843.1 & 1953 & AE009948.1 \\
\hline S. acidominimus & 1334 & X58301.1 & 373 & AJ511681.1 & 967 & AB239170.1 & 900 & AB238609.1 \\
\hline S. anginosus & 1559 & NR_041722.1 & 370 & AJ511731.1 & 971 & AB238697.1 & 900 & AB236189.1 \\
\hline S. alactolyticus & 1501 & EŪ̄28776.1 & 376 & AJ511706.1 & - & - & - & - \\
\hline S. australis & 1471 & NR_036936.1 & - & - & 648 & GU907541.1 & 458 & EU003771.1 \\
\hline S. caballi & 1430 & NR_044190.1 & - & - & - & - & - & - \\
\hline S. canis & 1482 & EU075058.1 & 374 & AJ511684.1 & 969 & AB238698.1 & 900 & AB236191.1 \\
\hline S. castoreus & 1508 & NR_042215.1 & - & - & - & - & 458 & EU003814.1 \\
\hline S. criceti & 1530 & EU4̄83241.1 & - & - & 964 & AB238699.1 & 900 & AB238610.1 \\
\hline S. cristatus & 1533 & NR_042771.1 & 373 & AJ511700.1 & 896 & AB238700.1 & 900 & AB238611.1 \\
\hline S. dentapri & 1550 & $\mathrm{AB} \overline{4} 69560.1$ & - & - & - & - & - & - \\
\hline S. dentirousetti & 1543 & NR_041460.1 & - & - & - & - & - & - \\
\hline S. devriesei & 1471 & EU4̄83245.1 & - & - & - & - & 457 & EU003742.1 \\
\hline S. didelphis & 1506 & AF176107.1 & - & - & 914 & AB238810.1 & 457 & EU003790.1 \\
\hline S. downei & 1542 & NR_042774.1 & 376 & AJ511699.1 & - & - & 900 & AB238618.1 \\
\hline S. dysgalactiae subsp equisimilis & 1492 & AP010935.1 & 374 & СР002215.1 & 1137 & AP010935.1 & 1953 & AP010935.1 \\
\hline S. constellatus & 1508 & AY277942.1 & 370 & AJ511742.1 & 964 & AB238808.1 & 509 & AB441109.1 \\
\hline S. intermedius & 1531 & GU470908.1 & 374 & AJ511743.1 & 547 & AB441138.1 & 1947 & AB562520.1 \\
\hline S. entericus & 1335 & NR_025500.1 & - & - & - & - & - & - \\
\hline S. equi subsp. equi & 1495 & FM204883.1 & 374 & FM204883.1 & 1137 & FM204883.1 & 1953 & FM204883.1 \\
\hline S. equinus & 1539 & AB362710.1 & 373 & AJ511745.1 & 916 & AB238704.1 & - & - \\
\hline S. ferus & 1540 & AB259060.1 & 366 & AJ511705.1 & 902 & AB238705.1 & 903 & AB238616.1 \\
\hline S. gallinaceus & 1502 & NR_025453.1 & - & - & - & - & 458 & EU003785.1 \\
\hline S. gallolyticus & 1535 & AF $\overline{3} 23911.1$ & 375 & AJ511683.1 & 1143 & AP012053.1 & 1953 & AP012053.1 \\
\hline S. gordonii str. Challis substr. CH1 & 1499 & СР000725.1 & - & - & 1134 & СР000725.1 & 1950 & СР000725.1 \\
\hline S. halichoeri & 1506 & NR_029025.1 & - & - & - & - & 458 & EU003791.1 \\
\hline S. henryi & 1430 & NR_044189.1 & - & - & - & - & - & - \\
\hline S. hyointestinalis & 1504 & EU7̄28763.1 & 372 & AJ511696.1 & 1014 & AB238709.1 & 900 & AB238621.1 \\
\hline S. hyovaginalis & 928 & $\begin{array}{l}\text { EF151158.1/ } \\
\text { DQ118670.1 }\end{array}$ & 376 & AJ512493.1 & 974 & AB238710.1 & 900 & AB238622.1 \\
\hline S. ictaluri & 1471 & DQ462421.1 & - & - & - & - & - & - \\
\hline S. infantarius subsp infantarius & 1494 & EU163504.1 & 371 & AJ511688.1 & 1008 & AB238812.1 & 458 & EU003810.1 \\
\hline S. infantis & 1468 & NR_042928.1 & 387 & AJ511687.1 & 835 & AB238711.1 & 900 & AB238623.1 \\
\hline S. iniae & 1536 & NR_025148.1 & 378 & AJ511708.1 & 905 & AB238712.1 & 900 & GU324259.1 \\
\hline S. luteciae & 1461 & NR_042051.1 & - & - & - & - & - & - \\
\hline S. lutetiensis & 1501 & JN71 3319.1 & 371 & AJ511709.1 & - & - & 458 & EU003729.1 \\
\hline S. macacae & 1542 & NR_042775.1 & 373 & AJ511702.1 & 1051 & AB238713.1 & 900 & AB238625.1 \\
\hline S. macedonicus & 1538 & $\mathrm{AF} \overline{4} 59431.1$ & 374 & AJ511677.1 & - & - & 458 & HE613569.1 \\
\hline S. marimammalium & 1500 & NR_025630.1 & - & - & - & - & 458 & EU003815.1 \\
\hline S. massiliensis & 1470 & NR_043173.1 & - & - & - & - & 458 & EU003813.1 \\
\hline S. merionis & 1354 & NR_042553.1 & - & - & - & - & - & - \\
\hline S. minor & 1497 & AY 232833.1 & - & - & - & - & 458 & EU003795.1 \\
\hline S. mitis & 1540 & AY518677.1 & 387 & AJ511694.1 & 1140 & FN568063.1 & 1947 & FN568063.1 \\
\hline S. oligofermentans & 1510 & NR_029052.1 & - & - & - & - & 458 & EU003768.1 \\
\hline S. oralis & 1470 & DQ232535.1 & 387 & AJ511698.1 & 1137 & FR720602.1 & 1947 & FR720602.1 \\
\hline S. orisratti & 1474 & EU075064.1 & 374 & AJ511692.1 & 1043 & AB238813.1 & 458 & EU003798.1 \\
\hline
\end{tabular}

Continued on next page 


\begin{tabular}{|c|c|c|c|c|c|c|c|c|}
\hline \multirow[t]{2}{*}{ Species } & \multicolumn{2}{|c|}{$16 \operatorname{SrRNA}$} & \multicolumn{2}{|r|}{$r n p B$} & \multicolumn{2}{|r|}{$d n a J$} & \multicolumn{2}{|r|}{$g y r B$} \\
\hline & $\mathrm{bp}$ & Accession No. & $\mathrm{bp}$ & Accession No. & $\mathrm{bp}$ & Accession No. & $\mathrm{bp}$ & Accession No. \\
\hline S. orisuis & 1553 & NR_041055.1 & - & - & - & - & - & - \\
\hline S. ovis & 1377 & NR_026471.1 & - & - & - & - & 458 & EU003799.1 \\
\hline S. parasanguinis & 1513 & NR_024842.1 & 373 & AJ511704.1 & 974 & AB238798.1 & 900 & СР002843.1 \\
\hline S. parauberis & 1541 & AF $\overline{2} 84579.2$ & 374 & СР002471.1 & 890 & AB238799.1 & 900 & AB238630.1 \\
\hline S. pasteurianus & 1511 & EU163495.1 & - & - & - & - & 458 & AP012054.1 \\
\hline S. peroris & 1329 & EU156772.1 & 387 & AJ511690.1 & 890 & AB238800.1 & 900 & AB238631.1 \\
\hline S. phocae & 1510 & HM032023.1 & 371 & AJ511670.1 & 935 & AB238801.1 & 900 & AB238632.1 \\
\hline S. pluranimalium & 1552 & Y18026.1 & 375 & AJ511697.1 & - & - & 458 & EU003801.1 \\
\hline S. plurextorum & 1451 & NR_042649.1 & - & - & - & - & - & - \\
\hline S. pneumoniae & 1511 & AM- 157442.1 & 386 & СР002121.1 & 1137 & AE005672.3 & 1947 & СР000918.1 \\
\hline S. porcinus & 1496 & NR_024634.1 & 369 & AJ511675.1 & - & - & 900 & $\mathrm{AB} 175052.1$ \\
\hline S. pseudopneumoniae & 1468 & СР002925.1 & - & - & - & - & 458 & EU003816.1 \\
\hline S. pseudoporcinus & 1491 & DQ303207.1 & - & - & - & - & - & - \\
\hline S. pyogenes & 1518 & FJ662846.1 & 374 & CP003121.1 & 1137 & AE009949.1 & 1953 & СР000262.1 \\
\hline S. ratti & 1444 & NR_025516.1 & 373 & AJ511671.1 & 896 & AB238804.1 & 900 & AB238635.1 \\
\hline S. salivarius & 1543 & AF $\overline{4} 59433.1$ & 373 & FR873482.1 & 621 & GU907550.1 & 900 & AB238640.1 \\
\hline S. sanguinis & 1510 & AY691542.1 & 373 & AJ511682.1 & 1134 & СР000387.1 & 1950 & CP000387.1 \\
\hline S. seminale & 1275 & AB370977.1 & - & - & - & - & - & - \\
\hline S. sinensis & 1512 & AF432855.1 & - & - & - & - & 458 & EU003809.1 \\
\hline S. sobrinus & 1551 & AB294731.1 & 373 & AJ511707.1 & - & - & 900 & AB238641.1 \\
\hline S. suis & 1536 & AF009497.1 & 370 & AJ511674.1 & 1137 & СР002633.1 & 1953 & СР002641.1 \\
\hline S. thermophilus & 1544 & EF990662.1 & 373 & AJ511712.1 & 1134 & FR875178.1 & 1953 & СР002340.1 \\
\hline S. thoraltensis & 1519 & NR_026368.1 & - & - & 885 & AB238806.1 & 900 & AB238637.1 \\
\hline S. uberis & 1501 & NR_040820.1 & 372 & AJ511693.1 & 1134 & AM946015.1 & 1953 & AM946015.1 \\
\hline S. urinalis & 1476 & DQ303194.1 & 373 & AJ511680.1 & - & - & 458 & EU003763.1 \\
\hline S. ursoris & 1549 & AB501126.1 & - & - & - & - & - & - \\
\hline S. vestibularis & 1553 & NR_042777.1 & 373 & AJ511724.1 & - & - & 900 & AB238643.1 \\
\hline L. lactis subsp Lactis & 1539 & СР001834.1 & - & - & 1140 & СР001834.1 & 1956 & СР001834.1 \\
\hline L. lactis subsp cremoris & 1539 & CP000425.1 & - & - & 1088 & СР000425.1 & 1956 & СР000425.1 \\
\hline Lactovum miscens & 1456 & NR_042140 & & & & & & \\
\hline Pilibacter termitis & 1432 & NR_042949 & & & & & & \\
\hline
\end{tabular}
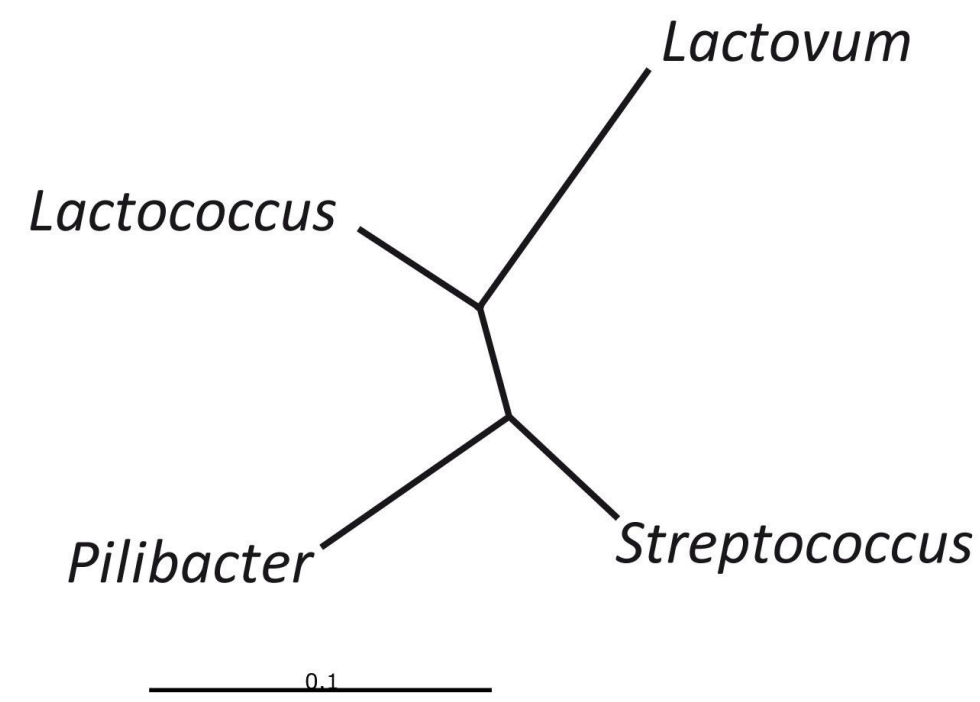

Figure 2. Unrooted tree obtained from a Bayesian analysis of representative species of the family Streptococcaceae, inferred from their 16sR RNA sequences (see details in Table 1). 


\section{RESULTS AND DISCUSSION}

Seven major clades were detected in our analyses; these corresponded to the conventional species groups, except for the group mitis that was split into two nonmonophyletic clades (Figure 3). In general, phylogenies inferred either from nucleotide or amino acid derived sequences did not differ either on major-clade relationships or statistical confidence (i.e., posterior probabilities).

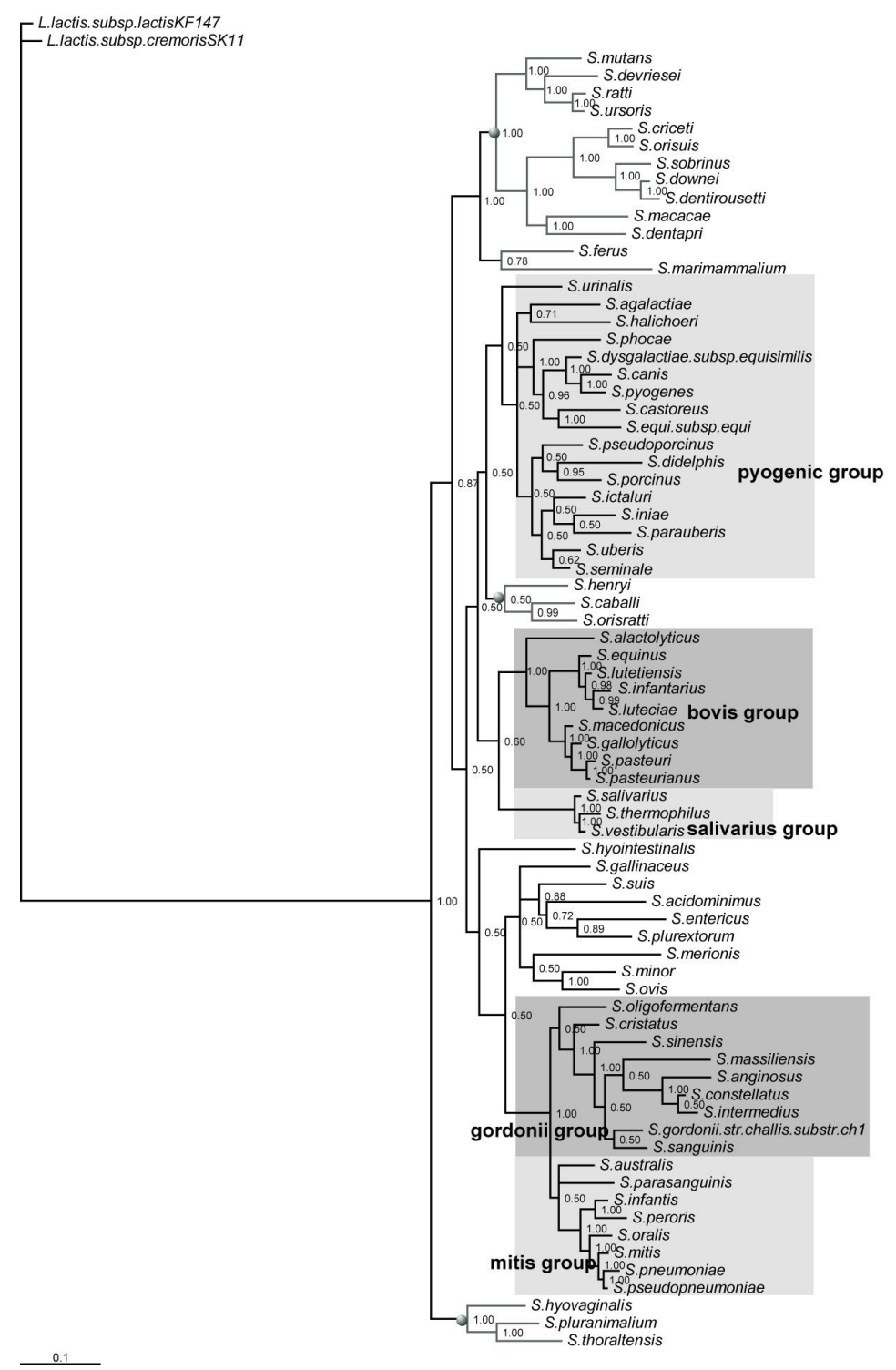

Figure 3. Majority consensus tree obtained by a Bayesian analysis of proteic (sodA, tuf, rpoB, dnaJ, gyrB) and nucleotidic (16sr RNA, rnpB) of the entire matrix. 
Table 2. Species of Streptococcus, and outgroup species, analyzed in this study.

\begin{tabular}{|c|c|}
\hline Mutans group & $\begin{array}{l}\text { S. ratti, } S \text {. ursoris, S. devriesei, } S . \text { mutans, } S . \text { macacae, S. ferus, } S . \text { dentapri, S. downei, S. dentirousetti, S. sobrinus, } \\
\text { S. dentirousetti, S. sobrinus, } S \text {. criceti, S. orisuis, S. merionis, S. caballi, S. henryi, S. orisratti, S. pluranimalium, } \\
\text { S. thoraltensis, S. hyovaginalis }\end{array}$ \\
\hline Pyogenic group & $\begin{array}{l}\text { S. dysgalactiae, S. pyogenes, S. canis, S. castoreus, S. equi subsp equi, S. halichoeri, S. phocae, S. porcinus, } \\
\text { S. pseudoporcinus, S. didelphis, S. uberis, S. seminale, S. iniae, S. ictaluri, S. urinalis, S. parauberis, } \\
\text { S. marimammalium, S. agalactiae }\end{array}$ \\
\hline Bovis group & S. equinus, $S$. lutetiensis, $S$. luteciae, $S$. infantarius, $S$. gallolyticus, $S$. pasteurianus, $S$. macedonicus, $S$. alactolyticus \\
\hline Suis group & S. entericus, $S$. plurextorum, $S$. suis, $S$. acidominimus, $S$. minor, $S$. ovis, $S$. gallinaceus \\
\hline Mitis group A & S. constellatus, $S$. intermedius, $S$. anginosus, $S$. massiliensis, $S$. cristatus, $S$. sinensis, $S$. gordonii, $S$. sanguinis \\
\hline Mitis group B & $\begin{array}{l}\text { S. pneumoniae, S. pseudopneumoniae, S. mitis, S. oligofermentans, S. infantis, S. peroris, S. oralis, } \\
\text { S. australis, S. parasanguinis }\end{array}$ \\
\hline Saliv & S. vestibularis, $S$. salivarius, $S$. thermophilus \\
\hline Outgroup & Lactococcus lactis, L. cremoris \\
\hline
\end{tabular}

With regard to the phylogenetic relationships within species groups, it is notable that the mitis group (Kawamura et al., 1995) remained monophyletic but, in agreement with the results of Boggs et al. (2012) (Figure 3), was clearly subdivided into two clades. The suis group was basal to the sister clades.

Here we propose a taxonomic revision, since our results indicate that divergence between the two major clades subdividing the group mitis is in the range of divergence for other species groups (within-mean distances for the "gordonii" and "mitis" clades were 0.039 and 0.044 , respectively; between-mean distance was 0.073 ). Therefore, a new species group was proposed and named gordonii; the group included the species $S$. constellatus, $S$. intermedius, S. anginosus [referred to as species group anginosus by Kawamura et al. (1995)], plus $S$. massiliensis, $S$. cristatus, $S$. sinensis, $S$. gordonii, and $S$. sanguinis. Additionally, the group mitis was retained, and included the species $S$. australis, $S$. parasanguinis, $S$. oralis, $S$. peroris, S. infantis, S. oligofermentans, S. mitis, S. pseudopneumoniae, and S. pneumoniae.

We suggest that gordonii is the basal group of the genus, followed by mitis, suis and finally pyogenic (Figure 3 ). The most recent divergence was the separation of the sister species groups salivarius and bovis. All the species groups, except mutans, resulted in monophyletic groups (Figure 3). S. ferus was basal to the entire genus, and not inside the mutans group as previously reported. Similarly, S. entericus and S. plurextorum (suis group), S. marimammalium and $S$. agalactiae (pyogenic group) were not placed in their previously described groups. The remaining species were clustered inside their formerly ascribed groups.

The diverse analyses performed here clearly show that the majority of the species groups had a stable topology. By changing the parameters of the gene sequences, i.e., using amino acid derived or nucleotide sequences, or by constraining clades, most of the species groups did not change their species composition. In the major part of the non-constrained analysis, the mutans species group resulted in a polyphyletic taxon, compressing at least three different lineages (Figure 3).

However, a homogeneity analysis revealed incompatibilities among gene sequences within the mutans species group; these incompatibilities might be due to horizontal gene transfer (Table 3) (Abascal et al., 2005; Itoh et al., 2006; Simmon et al., 2008; Boggs et al., 2012). More critical incompatibilities, detected by pairwise analysis, allowed us to identify large genetic discordances between different loci. We took these results into account in subsequent analyses, avoiding incompatibilities and generating different strategies in order to infer phylogenetic relationships within each species group and among groups. 
Table 3. Homogeneity test results. P values for all the pairwise combinations of gene and protein fragments are shown for each species group and for the "mini matrix" set of species.

\begin{tabular}{|c|c|c|c|c|c|c|c|}
\hline & Mutans group & Pyogenic group & Bovis group & Suis group & Mitis group A & Mitis group B & Mini matrix \\
\hline All & 0.01 & 0.01 & 0.27 & 0.88 & 0.01 & 0.01 & 0.01 \\
\hline sodA $x$ tuf & 0.26 & 0.12 & 0.56 & 0.99 & 0.14 & 0.01 & 0.04 \\
\hline sodA $x$ rpoB & 0.80 & 0.42 & 0.94 & 0.96 & 0.71 & 0.01 & 0.23 \\
\hline sodA x $16 s r$ & 0.02 & 0.01 & 0.30 & 0.53 & 0.17 & 0.01 & 0.03 \\
\hline sodA $x \operatorname{rnpB}$ & 0.01 & 0.58 & 0.05 & 0.97 & 0.42 & 0.01 & 0.16 \\
\hline sodA $x$ dnaJ & 0.01 & 0.61 & 0.93 & 0.99 & 0.68 & 0.39 & 0.01 \\
\hline sodA x gyrB & 0.27 & 0.04 & 0.88 & 0.38 & 0.62 & 0.03 & 0.93 \\
\hline sodA $x$ recN & 0.01 & 0.43 & 1 & 0.06 & 0.31 & 0.01 & 0.31 \\
\hline tuf $x$ rpoB & 0.41 & 0.37 & 1 & 1 & 0.51 & 0.01 & 0.02 \\
\hline tuf $\times 16 \mathrm{sr}$ & 0.28 & 0.45 & 0.38 & 0.98 & 0.07 & 0.02 & 0.04 \\
\hline tuf $x$ rnpB & 0.01 & 0.99 & 0.56 & 1 & 0.72 & 0.01 & 0.01 \\
\hline tuf $x$ dnaJ & 0.01 & 0.83 & 1 & 1 & 0.77 & 0.50 & 0.01 \\
\hline tuf $\mathrm{x}$ gyrB & 0.87 & 0.40 & 0.98 & 1 & 0.03 & 0.05 & 0.50 \\
\hline tuf $x$ recN & 0.01 & 0.83 & 0.89 & 0.97 & 0.26 & 0.01 & 0.23 \\
\hline rpoB x 16sr & 0.41 & 0.08 & 0.74 & 0.66 & 0.51 & 0.07 & 0.01 \\
\hline rpoB x rnpB & 0.17 & 0.91 & 1 & 1 & 0.35 & 0.31 & 0.01 \\
\hline rpoB x dnaJ & 0.03 & 0.78 & 1 & 1 & 0.21 & 0.28 & 0.03 \\
\hline rpoB x gyrB & 0.99 & 0.60 & 1 & 0.72 & 0.49 & 0.65 & 0.47 \\
\hline rpoB $x$ recN & 0.22 & 0.90 & 0.06 & 0.12 & 0.19 & 0.79 & 0.07 \\
\hline $16 s r \times$ rnpB & 0.05 & 0.16 & 0.49 & 0.98 & 0.01 & 0.05 & 0.09 \\
\hline $16 s r \times$ dnaJ & 0.10 & 0.29 & 0.92 & 1 & 0.69 & 0.14 & 0.01 \\
\hline $16 \mathrm{sr} x$ gyrB & 0.04 & 0.01 & 0.76 & 0.85 & 0.54 & 0.24 & 0.59 \\
\hline $16 \mathrm{sr} \times \mathrm{recN}$ & 0.01 & 0.01 & 0.88 & 0.41 & 0.68 & 0.01 & 0.33 \\
\hline rnpB x dnaJ & 0.29 & 1 & 1 & 1 & 0.01 & 0.94 & 0.01 \\
\hline rnpB x gyrB & 0.02 & 0.42 & 0.87 & 1 & 0.35 & 0.39 & 0.07 \\
\hline rnpB $x$ recN & 0.01 & 0.85 & 0.03 & 0.95 & 0.06 & 1 & 0.01 \\
\hline dnaJ x gyrB & 0.01 & 0.67 & 1 & 1 & 0.09 & 0.31 & 0.02 \\
\hline dnaJ $x$ recN & 0.01 & 0.80 & 1 & 0.99 & 0.01 & 0.56 & 0.01 \\
\hline gyrB x recN & 0.01 & 0.77 & 0.98 & 1 & 0.25 & 0.59 & 0.87 \\
\hline
\end{tabular}

For example, with regard to the mutans group, only $\operatorname{sod} A$, tuf, $r p o B$, and gyrB could be jointly analyzed, because only these genes shared high homogeneity scores (Table 3). Further analysis of this clade considered the entire matrix with the concatenated sequences of $\operatorname{sod} a, r p o B$, and $\operatorname{gyr} B$. These gene sequences were used in a new sampling strategy, which included all the species from the mutans group, plus two species representing each of the other species groups.

Next, we focused on the unsolved part of the tree: the mutans group and its relationship with salivarius, bovis, and pyogenic groups, using the same gene sequences and parameters as described above. This analysis reinforced the conclusion that the mutans group was not a monophyletic taxon. Rather, it appeared to be constituted by at least three lineages, namely mutans, sobrinus, and pluranimalium. This Bayesian tree resolved most of the relationships between these species (Figure 4).

Subsequently, we focused on unresolved parts of the phylogeny, namely, the relationship of $S$. macacae to the mutans and pyogenic groups, and the relationship between the pluranimalium and sobrinus groups. Homogeneity tests allowed us to select pairs of gene sequences to analyze for this part of the phylogeny (Table 4).

Bayesian analyses of tuf-recN rpoB-recN gene sequences placed $S$. macacae inside the mutans group, with $S$. ferus, S. mutans, $S$. devriesei, and $S$. ratti (Figure 5a). Bayesian trees of sodA$r p o B, 16 \mathrm{~S}$ rRNA-rnpB, and $\operatorname{sod} A-r e c N$ gene sequences identified these two groups as sister clades (Figure 5b). Nonetheless, the phylogenetic relationships of $S$. caballi and $S$. orisratti remained unsolved since insufficient nucleotide data were present in the databases. Moreover, there was a lack of phylogenetic signal due to the low rate of nucleotide substitution (i.e., 16S rRNA) and phylogenetic incompatibility between the gene sequences (i.e. between $16 \mathrm{~S}$ rRNA and $\operatorname{sod} A$ ). 


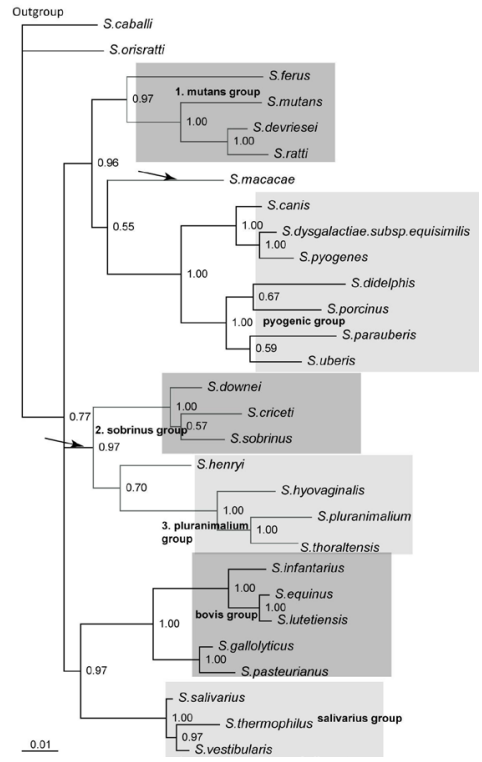

Figure 4. Majority consensus tree obtained from a Bayesian analysis of concatenated sequences of a subtree of Streptococcus using 3 proteic fragments ( $\operatorname{sod} \mathrm{A}, \mathrm{rpoB}, \mathrm{gyrB}$ ). Arrows indicate unsolved points of the topology. Species groups suis, gordonii and mitis are excluded from this subtree. The models of protein evolution were WAG (sodA) and Rtrev (rpoB, recN).

Table 4. Homogeneity test results. P values for all the pairwise combinations of gene and protein fragments are shown for each one of the two pairs of minigroups derived from the mutans group.

\begin{tabular}{|c|c|c|}
\hline & Clade A & Clade B \\
\hline sodA $x$ tuf & 0.56 & 0.14 \\
\hline sodA x rpoB & 0.98 & 0.87 \\
\hline sodA x $16 \mathrm{~s}$ & 0.72 & 0.01 \\
\hline sodA x rnpB & 0.94 & 0.01 \\
\hline sodA x dnaJ & 0.97 & 0.13 \\
\hline sodA x gyrB & 0.54 & 0.31 \\
\hline sodA x recN & 0.98 & 0.56 \\
\hline tuf $x$ rpoB & 0.92 & 0.68 \\
\hline tuf $\times 16 \mathrm{~s}$ & 0.75 & 0.63 \\
\hline tuf $\mathrm{x}$ rnpB & 1 & 0.14 \\
\hline tuf $x$ dna $J$ & 1 & 0.55 \\
\hline tuf $x$ gyrB & 0.90 & 0.33 \\
\hline tuf $x$ recN & 1 & 0.95 \\
\hline rpoB x $16 \mathrm{~s}$ & 0.95 & 0.02 \\
\hline rpoB x rnpB & 1 & 0.04 \\
\hline rpoB x dnaJ & 1 & 0.57 \\
\hline rpoB x gyrB & 1 & 0.56 \\
\hline $\mathrm{rpoB} \times \mathrm{recN}$ & 0.85 & 0.94 \\
\hline $16 \mathrm{~s} \times \mathrm{rnpB}$ & 0.98 & 0.08 \\
\hline $16 \mathrm{~s} \times \mathrm{dnaJ}$ & 0.96 & 0.06 \\
\hline 16 s x gyrB & 0.54 & 0.01 \\
\hline $16 \mathrm{~s} \times \mathrm{recN}$ & 0.92 & 0.01 \\
\hline rnpB x dnaJ & 1 & 0.08 \\
\hline rnpB x gyrB & 0.87 & 0.01 \\
\hline $\operatorname{rnpB} \times \mathrm{recN}$ & 1 & 0.57 \\
\hline dnaJ x gyrB & 1 & 0.06 \\
\hline $\mathrm{dnaJ} \times \mathrm{recN}$ & 1 & 0.78 \\
\hline gyrB x recN & 0.94 & 0.79 \\
\hline
\end{tabular}

Clade A = Streptococcus pluranimalium, S. thoraltensis, S. hyovaginalis, S. henryi, and S. criceti, S. sobrinus, $S$. downei. Clade B = S. devriesei, S. ratti, S. mutans, S. ferus, S. macacae, S. canis, S. pyogenes, $S$. dysgalactiae, $S$. didelphis, S. porcinus, and S. uberis. 


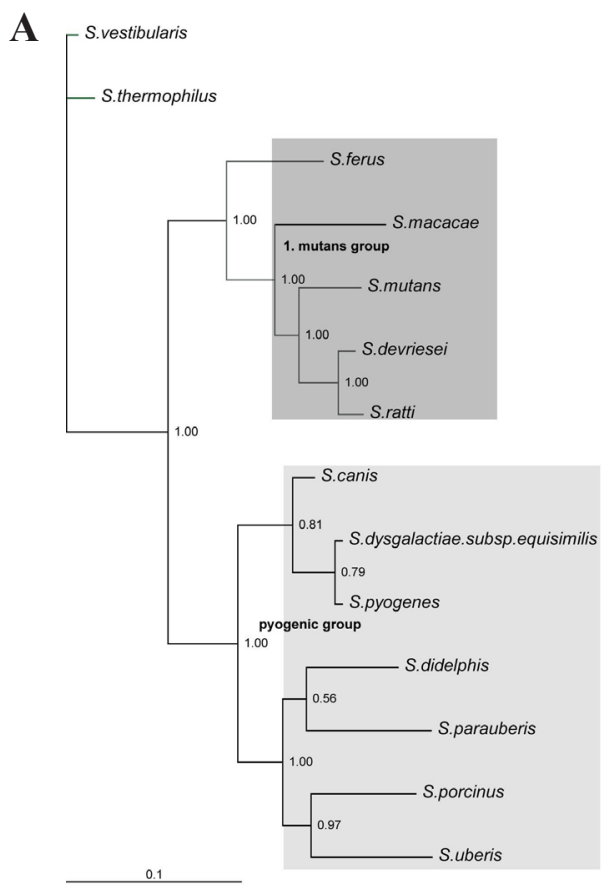

B

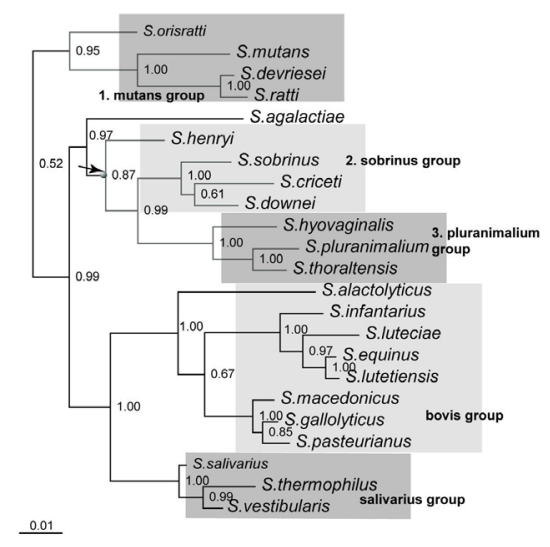

Figure 5. Majority consensus tree obtained of a Bayesian analysis of concatenated sequences. Those matrices were built using (A) 2 aminoacidic fragments ( $\mathrm{rpoB}$, recN), in order to solve the phylogenetic position of Streptococcus macacae; (B) 2 aminoacidic fragments (sodA, recN) to infer the relationship between sobrinus and pluranimalium groups. The models of protein evolution were WAG ( $\operatorname{sodA})$, Rtrev (rpoB), and Jones (recN).

We performed a phylogenetic analysis of a mini-matrix comprising two species from each of the nine groups (mutans, sobrinus, pluranimalium, salivarius, bovis, pyogenic, gordonii, mitis, and suis; Figure 6). The results showed that, in contrast to previous studies, the suis, gordonii, and mitis groups were basal clades. In contrast, sobrinus and pluranimalium were sister clades and closely related to the salivarius group. This phylogeny is shown in Figure 7, and represents our hypothesis on the phylogenetic relationships among the species groups of Streptococcus. 


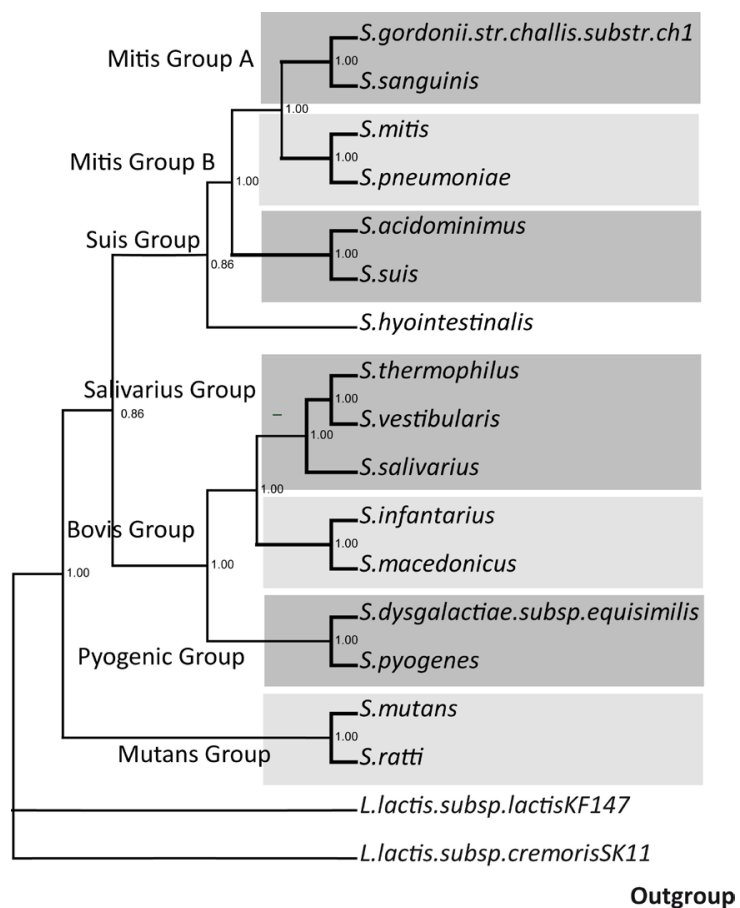

Figure 6. Majority consensus tree obtained by a Bayesian analysis of proteic ( $\operatorname{sod} A$, rpoB, and gyrB) matrix of the summarized tree containing two members of each group, plus the outgroup.

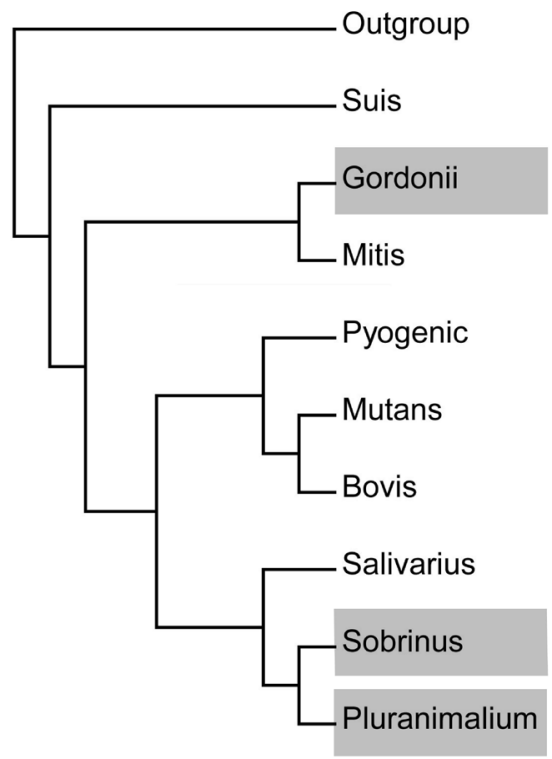

Figure 7. Hypothesis suggested by this study, summarizing the results obtained from the complete super-matrix and the mini-matrix analyses. 


\section{Conflicts of interest}

The authors declare no conflict of interest.

\section{ACKNOWLEDGMENTS}

Research supported by grants FONDECYT \#1110461, FONDECYT \#1140544, VIDEnlace \#70219 and Programa de Investigación Asociativa Conicyt, Anillo \#ACT-096.

\section{REFERENCES}

Abascal F, Zardoya R and Posada D (2005). ProtTest: selection of best-fit models of protein evolution. Bioinformatics 21: 2104-2105.

Boggs JM, South AH and Hughes AL (2012). Phylogenetic analysis supports horizontal gene transfer of L-amino acid oxidase gene in Streptococcus oligofermentans. Infect. Genet. Evol. 12: 1005-1009.

Facklam R (2002). What happened to the streptococci: overview of taxonomic and nomenclature changes. Clin. Microbiol. Rev. 15: 613-630.

Farris JS, Källersjö M, Kluge AG and Bult C (1994). Testing significance of incongruence. Cladistics 10: 315-319.

Farris JS, Källersjö M, Kluge AG and Bult C (1995). Constructing a significance test for incongruence. Syst. Biol. 44: 570-572.

Glazunova OO, Raoult D and Roux V (2006). Streptococcus massiliensis sp. nov., isolated from a patient blood culture. Int. J. Syst. Evol. Microbiol. 56: 1127-1131.

Glazunova OO, Raoult D and Roux V (2010). Partial recN gene sequencing: a new tool for identification and phylogeny within the genus Streptococcus. Int. J. Syst. Evol. Microbiol. 60: 2140-2148.

Hall TA (1999). BioEdit: a user-friendly biological sequence alignment editor and analysis program for Windows 95/98/ NT. Nucl. Acids Symp. Ser. 41: 95-98.

Hardie JM and Whiley RA (1997). Classification and overview of the genera Streptococcus and Enterococcus. Soc. Appl. Bacteriol. Symp. Ser. 83: 1S-11S.

Itoh Y, Kawamura Y, Kasai H, Shah MM, et al. (2006). dnaJ and gyrB gene sequence relationship among species and strains of genus Streptococcus. Syst. Appl. Microbiol. 29: 368-374.

Kawamura Y, Hou XG, Sultana F, Miura H, et al. (1995). Determination of 16S rRNA sequences of Streptococcus mitis and Streptococcus gordonii and phylogenetic relationships among members of the genus Streptococcus. Int. J. Syst. Bacteriol. 45: 406-408.

Lal D, Verma M and Lal R (2011). Exploring internal features of 16S rRNA gene for identification of clinically relevant species of the genus Streptococcus. Ann. Clin. Microbiol. Antimicrob. 10: 28.

Montes M and García-Arenzana JM (2007). Género Streptococcus: una revisión práctica para el laboratorio de microbiología. Enferm. Infecc. Microbiol. Clin. 25: 14-20.

Nylander JAA (2004). MrModeltest v2. Program distributed by the author. Evolutionary Biology Centre. Uppsala University.

Posada D and Crandall KA (1998). MODELTEST: testing the model of DNA substitution. Bioinformatics 14: 817-818.

Rodicio M and Mendoza MC (2004). Identificación bacteriana mediante secuenciación del ARNr 16S: fundamento, metodología y aplicaciones en microbiología clínica. Enferm. Infecc. Microbiol. Clin. 22: 238-245.

Simmon KE, Hall L, Woods CW, Marco F, et al. (2008). Phylogenetic analysis of viridans group streptococci causing endocarditis. J. Clin. Microbiol. 46: 3087-3090.

Täpp J, Thollesson M and Herrmann B (2003). Phylogenetic relationships and genotyping of the genus Streptococcus by sequence determination of the RNase P RNA gene, rnpB. Int. J. Syst. Evol. Microbiol. 53: 1861-1871.

Wiens JJ and Moen DS (2008). Missing data and the accuracy of Bayesian phylogenetics. J. Syst. Evol. 46: 307-314. 